\title{
Study of the Longitudinal Edge Effect \\ in Bilateral Linear Induction Machines \\ with Liquetallic Rotor
}

\author{
Ivan V. Kizhaev*, \\ Viktor N. Timofeev and Maxim Yu. Khatsayuk \\ Siberian Federal University \\ 79 Svobodny, Krasnoyarsk, 660041, Russia
}

Received 11.10.2018, received in revised form 31.10.2018, accepted 10.01.2019

This article analyzes the electromagnetic field of a flat linear induction machine with an analytical method of mathematical modeling. Graphs of the electromagnetic characteristics of the machines were obtained, taking into account the longitudinal edge effect at various values of slip and coefficients of quality factor. Also made a comparative assessment of the effect of the longitudinal edge effect on the performance of the machine.

Keywords: MHD pump, integral and differential characteristics, analytical calculation.

Citation: Kizhaev I.V., Timofeev V.N., Khatsayuk M.Yu. Study of the longitudinal edge effect in bilateral linear induction machines with liquetallic rotor, J. Sib. Fed. Univ. Eng. technol., 2019, 12(2), 240-249. DOI: 10.17516/1999-494X-0133.

\section{Исследование продольного краевого эффекта}

\section{в двухсторонних линейных индукционных машинах с жидкометаллическим ротором}

\author{
И.В. Кижаев, В.Н. Тимофеев, М.Ю. Хацаюк \\ Сибирский федеральный университет \\ Россия, 660041, Красноярск, пр. Свободный, 79
}

В данной статье проведен анализ электромагнитного поля плоской линейной индукционной машины с помощью аналитического метода математического моделирования. Получены графики электромагнитных характеристик машины с учетом продольного краевого эффекта при различных величинах скольжения и коэффициента добротности. Также проведена сравнительная оченка влияния продольного краевого эффекта на показатели машины.

(C) Siberian Federal University. All rights reserved

This work is licensed under a Creative Commons Attribution-NonCommercial 4.0 International License (CC BY-NC 4.0).

* Corresponding author E-mail address: ivan333x@mail.ru 
Ключевые слова: МГД-насос, интегральные и дифференциальные характеристики, аналитический расчет.

\section{Введение}

В настоящее время перспективным направлением в области энергетики является атомная промышленность. С ростом мощностей атомных электростанций растут требования к оборудованию, осуществляющему технологические процессы. Основой оборудования служат системы перекачивания жидкого теплоносителя. В них используются магнитогидродинамические (МГД) насосы индукционного и кондукционного типов, в зависимости от условий эксплуатации. Использование кондукционных насосов не всегда представляется возможным из-за многих факторов, один из которых перекачивание агрессивных высокотемпературных сред. Следовательно, приходится прибегать к линейным индукционным МГД-насосам, основным недостатком которых считают продольный и поперечный краевые эффекты.

Конечная длина индукторов линейных индукционных машин (ЛИМ) обуславливает появление в возбуждающем поле кроме бегущей волны две стоячие пульсирующие волны. При наличии в зазоре вторичного проводящего и движущегося тела в последнем возникают не только ответные бегущие волны тока и поля, но и дополнительные токи и поля, оказывающие, как правило, тормозное действие, увеличивающие потери и снижающие к. п. д. машины в целом. В некоторых случаях вредное влияние продольного краевого эффекта может быть очень значительным.

Проблеме изучения продольного краевого эффекта посвящено большое количество работ, одна из них [1]. Обычно на современном этапе делаются попытки решения двух- и трехмерных задач, что неизбежно приводит к численному анализу и чрезвычайно затрудняет понимание физики явления и его особенностей. Поэтому представляется целесообразным рассматривать одномерную модель с такими упрощениями, которые минимально искажают реальную физическую картину явления и в то же время позволяют максимально упростить математическое решение задачи. С этой целью мы воспользуемся расчетной моделью, предложенной и проанализированной А. И. Вольдеком [2, 3].

\section{Расчетная модель и принятые допущения}

Эскиз плоской линейной индукционной машины (ПЛИН) представлен на рис. 1 и включает в себя: 1 - магнитопроводы; 2 - многофазные обмотки; 3 - стенку канала толщиной $\chi$; 4 - жидкий металл, движущийся со скоростью $v$. Индукторы расположены на расстоянии $\delta$ от центра канала с жидким металлом и имеют длину $L=2 p \tau$, где $p$ - число пар полюсов; $\tau$ - полюсное деление.

Для анализа электромагнитных характеристик были приняты следующие основные допущения:

1. Магнитопроводы индукторов имеют магнитную проницаемость $\mu=\infty$ и электропроводность $\gamma=0$.

2. Поперечный краевой эффект отсутствует, т.е. ширина индукторов по оси $у$ не ограничена.

3. Система имеет бесконечную длину в направлении оси $y$, т.е. электромагнитное поле является плоскопараллельным. Вектор комплексной напряженности электрического поля $\dot{\bar{E}}=\bar{e}_{y} \dot{E}_{y}$,

$$
-241-
$$




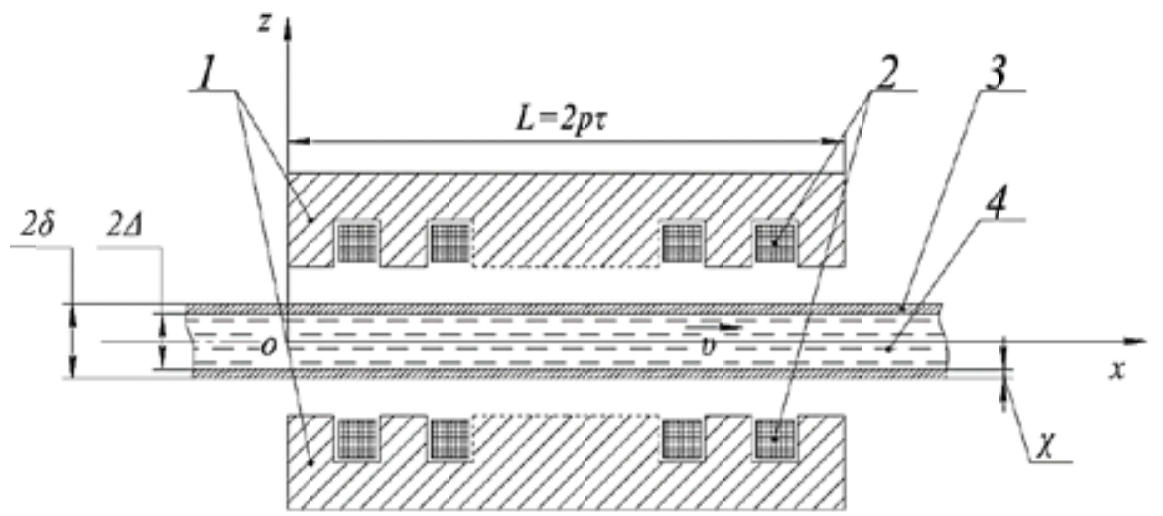

Рис. 1. Эскиз плоской линейной индукционной машины

Fig. 1. Sketch of a flat linear induction machine

вектор комплексной напряженности магнитного поля $\dot{\bar{H}}=\bar{e}_{x} \dot{H}_{x}+\bar{e}_{z} \dot{H}_{z}$, где $\bar{e}_{x}, \bar{e}_{y}, \bar{e}_{z}-$ единичные векторы прямоугольной системы координат.

4. Систему координат принимаем жестко связанной с индукторами, при этом все элементы жидкого металла в канале двигаются относительно индукторов с одинаковой скоростью v.

5. Влияние пазов учитывается обмотками, представленными бесконечно тонкими токовыми слоями, расположенными на поверхности индуктора с комплексной линейной плотностью тока:

$$
\dot{J}_{m}=J e^{i \varphi_{m}}
$$

где $J$-линейная плотность тока; $i=\sqrt{-1}$ - мнимая единица; $\varphi_{m}-$ начальный угол фазы $m$.

При принятых допущениях расчетная модель будет иметь вид, изображенный на рис. 2.

\section{Математическая модель линейной индукционной машины}

Решение будем проводить в двух областях: $1-0 \leq z<\Delta ; 2-\Delta<z \leq \delta$. Для напряженности электрического поля справедливы дифференциальные уравнения.

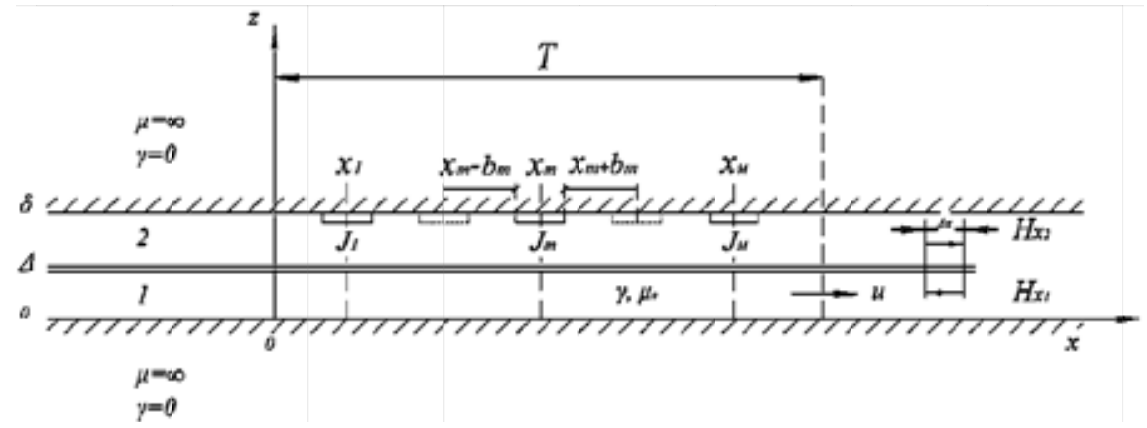

Рис. 2. Расчетная модель МГД-насоса

Fig. 2. MHD-pump design model 
Для области $1,0 \leq z<\Delta$ :

$$
\frac{\partial^{2} \dot{E}_{1}}{\partial z^{2}}+\frac{\partial^{2} \dot{E}_{1}}{\partial x^{2}}-i \mu_{0} \gamma \omega \dot{E}_{1}-\mu_{0} \gamma u \frac{\partial^{2} \dot{E}_{1}}{\partial x}=0
$$

где $\mu 0=4 \pi 10^{-7}[\Gamma \mu / M]-$ магнитная постоянная; $\gamma[O M / M]-$ удельное сопротивление; $\omega=2 \pi f$ $[p a d / c]$ - угловая частота; $u[\mu / c]-$ скорость движения жидкого металла.

Для области $2, \Delta<z \leq \delta$ :

$$
\frac{\partial^{2} \dot{E}_{2}}{\partial z^{2}}+\frac{\partial^{2} \dot{E}_{2}}{\partial x^{2}}=0
$$

Граничные условия:

$$
\begin{aligned}
& \left.\frac{\partial^{2} \dot{E}_{1}}{\partial z}\right|_{z=0}=0 ; \\
& \left.\frac{\partial^{2} \dot{E}_{2}}{\partial z}\right|_{z=\delta}=\left\{\begin{array}{c}
-i \omega \mu_{0} \dot{J}_{m} ; x_{m}-b_{m}<x<x_{m}+b_{m} \\
0
\end{array}\right. \\
& \frac{\partial \dot{E}_{2}}{\partial z}-\frac{\partial \dot{E}_{1}}{\partial z}=\left.i \omega \mu_{0} \sigma \chi E_{2}\right|_{z=\Delta},
\end{aligned}
$$

где $\sigma[C M]$ - удельная проводимость; $\chi[M]$ - толщина стенки канала.

\section{Решение дифференциальных уравнений}

Полагая, что электромагнитное поле периодично по координате $x$ с периодом $T$, будем искать решения в виде рядов Фурье в комплексной форме [4]:

$$
\begin{aligned}
& \dot{E}_{1,2}=\sum_{n=-\infty} \dot{E}_{1,2 n} e^{i k_{n} x} \\
& \dot{E}_{1,2 n}=\frac{1}{T} \int_{0}^{T} \dot{E}_{1,2} e^{-i k_{n} x} \partial x
\end{aligned}
$$

Умножив уравнения (2) и (3) на функцию $e^{-i k_{n} x}$ и проинтегрировав получившееся выражение по $x$ от 0 до $T$, получим:

$$
\begin{aligned}
& \frac{d^{2} \dot{E}_{1}}{d z^{2}}-\varphi_{n}^{2}=0 ; \\
& \frac{d^{2} \dot{E}_{2}}{d z^{2}}-k_{n}^{2} \dot{E}_{2}=0 ;
\end{aligned}
$$

где $\varphi_{n}=\sqrt{k_{n}^{2}+i \mu_{0} \gamma \omega+i k_{n} \mu_{0} \gamma u} \tau ; k_{n}=\frac{2 n \pi}{\tau}$.

Общими решениями обыкновенных дифференциальных уравнений (9) и (10) являются:

$$
\begin{aligned}
& \dot{E}_{1 n}=A_{1} e^{\varphi_{n} z}+A_{2} e^{-\varphi_{n} z} ; \\
& \dot{E}_{2 n}=A_{3} e^{k_{n} z}+A_{4} e^{-k_{n} z} .
\end{aligned}
$$

Постоянные интегрирования $A_{1} \ldots A_{4}$ определяются из граничных условий (4-6). 
Подставив полученные постоянные интегрирования из граничных условий в (11) и (12), получим:

$$
\begin{aligned}
& \dot{E}_{1 n}(z)=\frac{\Psi_{n} \operatorname{ch} \varphi_{n} z}{Q_{n}} \\
& \dot{E}_{2 n}(z)=\frac{\Psi_{n}}{k_{n}} \frac{\theta_{n}(z)}{Q_{n}}
\end{aligned}
$$

где $Q_{n}=k_{n} \operatorname{ch} \varphi_{n} \Delta s h k_{n}(\delta-\Delta)+G_{n} \operatorname{ch} k_{n}(\delta-\Delta)$;

$$
\begin{aligned}
& \theta_{n}(z)=k_{n} \operatorname{ch} \varphi_{n} \Delta \operatorname{ch} k_{n}(z-\Delta)+G_{n} \operatorname{shk}_{n}(z-\Delta) ; \\
& G_{n}=\varphi_{n} \operatorname{sh} \varphi_{n} \Delta+\xi \operatorname{ch} \varphi_{n} \Delta ; \\
& \xi=i \varepsilon_{k} \chi \pi^{2} \\
& \varepsilon_{k}=\frac{\omega \mu_{0} \sigma_{k}}{\pi^{2}} \tau^{2} .
\end{aligned}
$$

Преобразовав (13) и (14), имеем:

$$
\begin{aligned}
& \dot{E}_{1 n}(x, z)=\sum_{n=-\infty}^{n=+\infty} \frac{\Psi_{n}}{Q_{n}} \operatorname{ch} \varphi_{n} z e^{i k_{n} x} \\
& \dot{E}_{2 n}(x, z)=\sum_{n=-\infty}^{n=+\infty} \frac{\Psi_{n}}{k_{n} Q_{n}} \theta_{n}(z) e^{i k_{n}}
\end{aligned}
$$

где $\Psi_{n}=-\frac{i \omega \mu_{0}}{T} \sum_{m=1}^{M} \dot{J}_{m} \int_{x_{m}-b_{m}}^{x_{m}+b_{m}} e^{-i k_{n} x} \partial x=-i \frac{2 \omega \mu_{0}}{k_{n} T} \sum_{m=1}^{M} \dot{J}_{m} \sin \left(k_{n} b_{m}\right) e^{-i k_{n} x_{m}}=-i \frac{2 \omega \mu_{0}}{k_{n} T} C_{n 1}$

$$
C_{n 1}=\sum_{m=1}^{M} J_{m} \sin \left(k_{n} b_{m}\right) e^{-i k_{n} x_{m}}
$$

Окончательно получим

$$
\begin{aligned}
& \dot{E}_{1 n}(x, z)=-i \frac{2 \omega \mu_{0}}{T} \sum_{n=-\infty}^{n=+\infty} \frac{c h \varphi_{n} z}{k_{n} Q_{n}} C_{n 1} e^{i k_{n} x} \\
& \dot{E}_{2 n}(x, z)=-i \frac{2 \omega \mu_{0}}{T} \sum_{n=-\infty}^{n=+\infty} \frac{C_{n 1}}{k_{n}^{2} Q_{n}} \theta_{n}(z) e^{i k_{n} x}
\end{aligned}
$$

По известным уравнениям электромагнитного поля определим составляющие вектора магнитной индукции. Из $\operatorname{rot} E=-i \omega B$ :

$$
\begin{aligned}
& \dot{B}_{x}=\frac{1}{i \omega} \frac{\partial E_{y}}{\partial z} ; \\
& \dot{B}_{z}=\frac{1}{i \omega} \frac{\partial E_{y}}{\partial x} .
\end{aligned}
$$

Преобразовав (19) и (20) в соответствии с (17) и (18), получим:

$$
\dot{B}_{x 1}=-\frac{2 \mu_{0}}{T} \sum_{n=-\infty}^{n=+\infty} \frac{\varphi_{n} \operatorname{sh} \varphi_{n} z}{k_{n} Q_{n}} C_{n 1} e^{i k_{n} x}
$$




$$
\begin{aligned}
& \dot{B}_{z 1}=-\frac{i 2 \mu_{0}}{T} \sum_{n=-\infty}^{n=+\infty} \frac{s h \varphi_{n} z}{Q_{n}} C_{n 1} e^{i k_{n} x} ; \\
& \dot{B}_{x 2}=-\frac{2 \mu_{0}}{T} \sum_{n=-\infty}^{n=+\infty} \frac{C_{n 1}}{k_{n} Q_{n}} \theta_{n}(z) e^{i k_{n} x} ; \\
& \dot{B}_{z 2}=-\frac{i 2 \mu_{0}}{T} \sum_{n=-\infty}^{n=+\infty} \frac{C_{n 1}}{k_{n} Q_{n}} \theta_{n}^{\prime}(z) e^{i k_{n} x},
\end{aligned}
$$

где $\theta_{n}^{\prime}(z)=P_{n} \operatorname{sh} k_{n}(z-\Delta)+G_{n} \operatorname{chk}_{n}(z-\Delta)$.

\section{Интегральные и дифференциальные характеристики}

\section{линейной индукционной машины}

Комплексная электромагнитная мощность, отдаваемая обмоткой индуктора в зазор, равна:

$$
\underline{S}_{e m}=-2 l \sum_{m=1}^{M} \int_{x_{m}-b_{x}}^{x_{m}+b_{x}} \dot{E}_{2}(x, \delta){ }^{*} J_{m} \partial x=\frac{i 8 \omega \mu_{0} l}{T} \sum_{n=-\infty}^{n=+\infty} \frac{C_{n 1} C_{n 2}}{k_{n}^{3} Q_{n}} \theta_{n}(\delta),
$$

где $C_{n 2}=\sum_{m=1}^{M}{\stackrel{J}{J_{m}}}_{\sin } \sin \left(k_{n} b_{m}\right) e^{i k_{n} x_{m}}$.

Электромагнитная сила, действующая на индуктор:

$$
F_{e m}=\operatorname{Re}\left\{2 l \sum_{m=1}^{M} \int_{x_{m}-b_{m}}^{x_{m}+b_{m}} \dot{B}_{z 2}(x, \delta) J_{m}^{*} \partial_{i}=\operatorname{Re}\left\{i \frac{8 \mu_{0} l}{T} \sum_{n=-\infty}^{\infty} \frac{C_{n 1} C_{n 2}}{k_{n}^{2} Q_{n}}\left[\theta_{n}(\delta)\right\} .\right.\right.
$$

Приведем мощность к безразмерному виду. Для начала домножим и разделим все величины, входящие в $\frac{\theta_{n}}{Q_{n}}$ на $\tau$, отсюда

где $\hat{T}=\frac{T}{\tau}$.

$$
k_{n}=\frac{2 n \pi}{T} \tau=\frac{2 n \pi}{\hat{T}}
$$

где $\varepsilon=\frac{\mu_{0} \gamma \omega}{\pi^{2}} \tau^{2}$

$$
\hat{\varphi}_{n}=\sqrt{\left(\frac{2 n \pi}{\hat{T}}\right)^{2}+i \varepsilon \pi^{2}+i \frac{2 n}{\hat{T}} \varepsilon \pi^{2}(1-s)},
$$

Преобразуем (25) в соответствии с полученными значениями:

$$
\underline{S}_{e m}=\frac{i \omega \mu_{0} l T^{2}}{\pi^{3}} J_{b}^{2} \sum_{n=-\infty}^{n=+\infty} \frac{\theta_{n}(\delta)}{n^{3} Q_{n}}{\hat{C_{n 1}}}^{*}{\hat{C_{n 2}}}^{*}
$$

где $\hat{C_{n 1}}=\sum_{m=1}^{M} \hat{J_{m}} \sin \left(k_{n} b_{m}\right) e^{-i k_{n} x_{m}} ; \hat{C_{n 2}}=\sum_{m=1}^{M} \hat{J_{m}^{*}} \sin \left(k_{n} b_{m}\right) e^{i k_{n} x_{m}} ; \hat{J_{m}}=\frac{J_{m}}{J_{b}} ;{\hat{J_{m}}}^{*}=\frac{J_{m}}{J_{b}} ; J_{b}=\sum_{m=1}^{M} \frac{w_{m} I_{m}}{2 p \tau}$.

Поделим $\underline{S}_{e m}$ на базисную мощность, чтобы привести ее к безразмерному виду. За базисную мощность примем

$$
\underline{S}_{b}=\frac{\omega \mu_{0} l T^{2}}{\pi^{3}} J_{b}^{2}
$$


Окончательно

$$
{\underline{S_{e m}}}^{\wedge}=i \frac{\underline{S}_{e m}}{\underline{S}_{b}}=i \sum_{n=-\infty}^{n=+\infty} \frac{\theta_{n}(\delta)}{n^{3} Q_{n}} \hat{C_{n 1}} \hat{C_{n 2}}
$$

\section{Анализ расчета электромагнитных характеристик}

Как следует из полученных выражений, безразмерные характеристики индукционной линейной машины определяют следующие параметры:

1. Длина расчетной области. Условие $T=2 \tau$ позволяет анализировать электромагнитные характеристики с учетом влияния продольного краевого эффекта.

2. Количество пазов $M$.

3. Амплитудно-фазовые характеристики синусоидальных токов в пазах $\dot{J}_{m}=J e^{i \varphi_{m}}$.

4. Относительная величина воздушного зазора $-\hat{\delta}-\hat{\Delta}$.

5. Безразмерный коэффициент добротности $\varepsilon=\mu_{0} \gamma \omega \tau^{2} / \pi^{2}$.

В ходе работы были получены следующие результаты:

На рис. $3 a$ представлены классические механические характеристики асинхронных машин при различных величинах добротности $\varepsilon$ без проявления продольного краевого эффекта. С учетом краевого эффекта картина распределения относительной активной мощности $\hat{P_{e m}}$ (рис. $\left.3 б\right)$ изменяется. Если в случае без учета продольного краевого эффекта (рис. $3 a$ ) с увеличением коэффициента добротности максимальное значение относительной активной мощности не меняется, то во втором случае (рис. 3б) происходит «заваливание» характеристик с последующим снижением максимального значения активной мощности.

Аналогично относительной активной мощности изменения произошли и с картиной распределения относительной реактивной мощности $\hat{Q_{e m}}$ с влиянием продольного краевого эф-
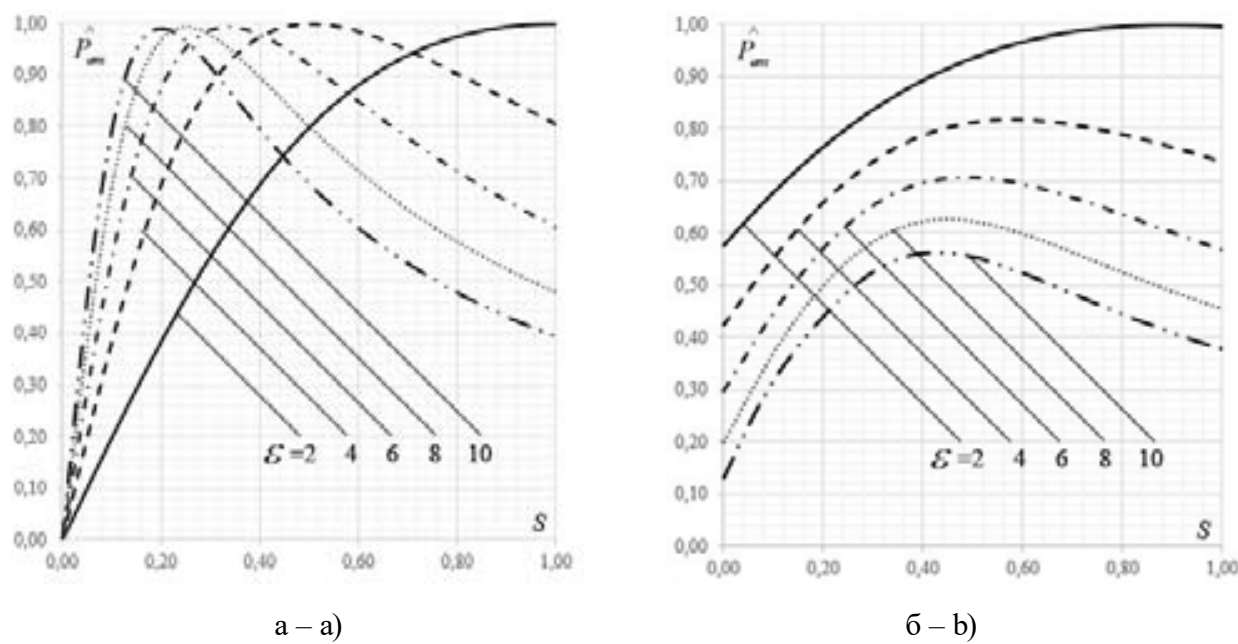

Рис. 3. График распределения относительной активной мощности относительно скольжения: a-без учета продольного краевого эффекта; б - с учетом влияния краевого эффекта

Fig. 3. Graph of relative active power from slip: a - without longitudinal edge effect; $b$ - with longitudinal edge effect 
фекта (рис. 4б). В данном случае графики исходят не из одной точки в отличие от классических характеристик асинхронных машин без учета краевого эффекта (рис. $4 a$ ).

Также были получены картины распределения относительной магнитной индукции $\hat{B}_{x 1}$ относительно длины расчетной области Т при различных скольжениях в непосредственной близости от стенки канала. В первом случае при $s=0,3$ на рис. $5 a$ графики магнитной индукции имеют вид обыкновенной замкнутой асинхронной машины. Вследствие конечной длины машины (рис. 5б) на графиках ярко выражено влияние продольного краевого эффекта в виде
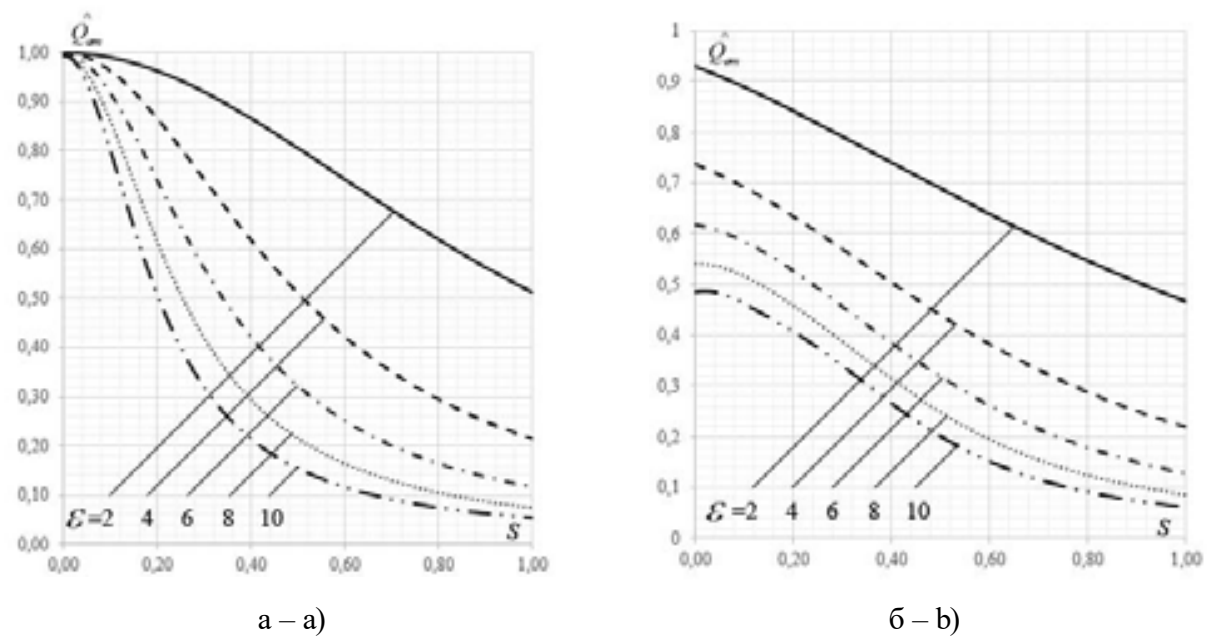

Рис. 4. График распределения относительной реактивной мощности относительно скольжения: a - без учета продольного краевого эффекта; б - с учетом влияния краевого эффекта

Fig. 4. Graph of relative reactive power from slip: a - without longitudinal edge effect; $b$ - with longitudinal edge effect
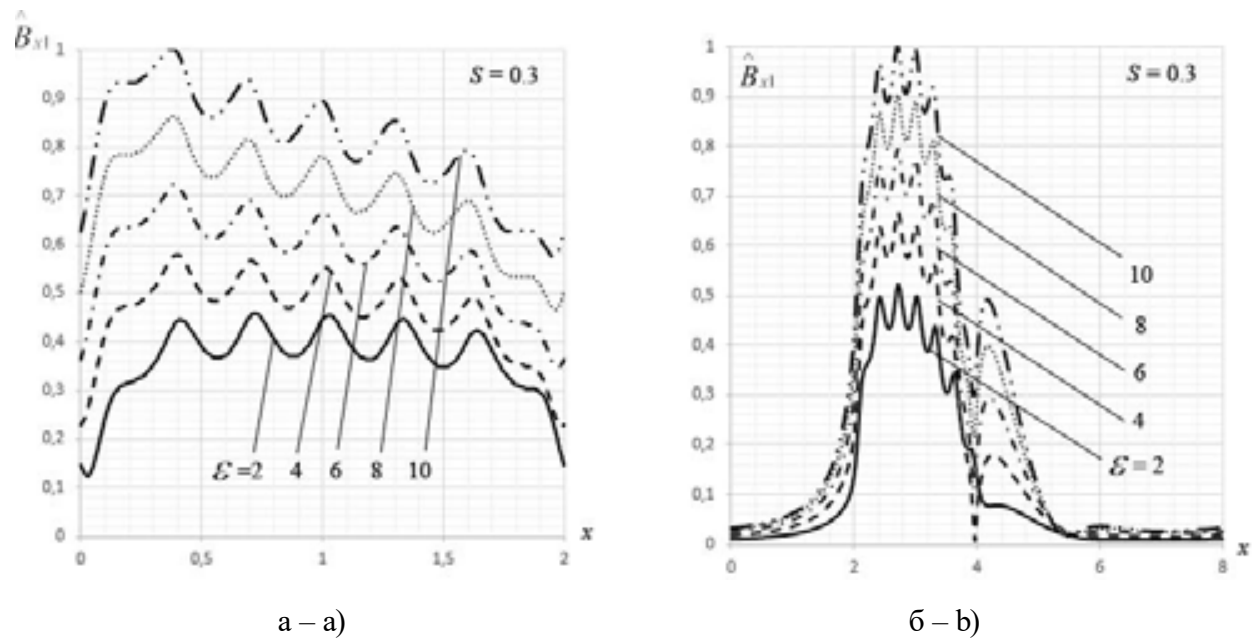

Рис. 5. График распределения относительной магнитной индукции относительно длины расчетной области: а - без учета продольного краевого эффекта; б - с учетом влияния краевого эффекта

Fig. 5. Graph of the distribution of relative magnetic induction from the length of the computational domain: a without longitudinal edge effect; $b$ - with longitudinal edge effect 


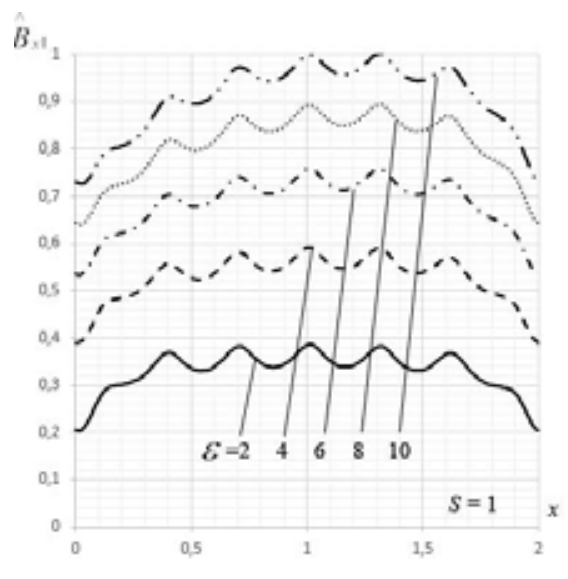

$\mathrm{a}-\mathrm{a})$

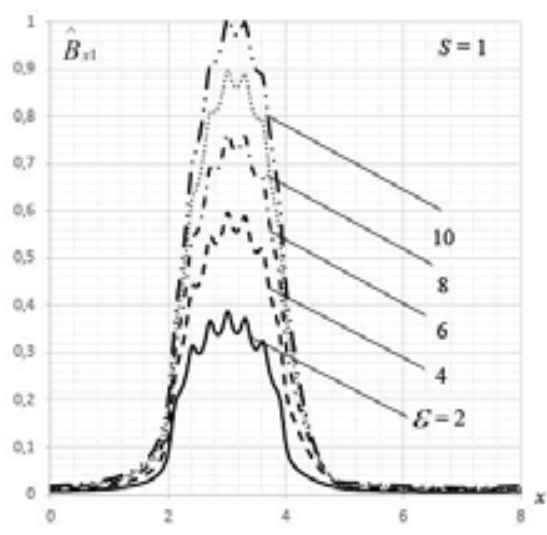

$6-b)$

Рис. 6. График распределения относительной магнитной индукции относительно длины расчетной области: а - без учета продольного краевого эффекта; б - с учетом влияния краевого эффекта

Fig. 6. Graph of the distribution of relative magnetic induction from the length of the computational domain: a without longitudinal edge effect; $b$ - with longitudinal edge effect

резкого снижения величины относительной магнитной индукции. При этом эффект тем более выражен, чем больше значение коэффициента добротности.

В случае, когда $s=0,3$ (рис. 6), такого ярковыраженного проявления продольного краевого эффекта не наблюдается даже при увеличении значения коэффициента добротности.

\section{Выводы}

С помощью преобразования с использованием рядов Фурье в комплексной форме решена двухмерная задача по анализу продольного краевого эффекта в двухсторонних линейных индукционных машинах. С помощью полученных выражений: векторов электромагнитного поля, мощностей и удельных электромагнитных сил в расплаве, можно анализировать электромагнитные характеристики линейных индукционных машин с влиянием продольного краевого эффекта.

Проведен анализ дифференциальных и интегральных характеристик двухсторонних линейных индукционных МГД-насосов. Получены зависимости относительных активной, реактивной мощности и магнитной индукции в зависимости от коэффициента добротности.

Исследование выполнено при финансовой поддержке РФФИ и Правительства Красноярского края в рамках научного проекта №18-48-242013 “Исследование влияния пространственных и частотно-временных распределений электромагнитных сил в расплаве на характер протекания МГД-процессов в жидком металле”.

\section{Список литературы}

[1] Штурман Г. И., Аронов Р. Л. Краевой эффект в индукционных машинах с разомкнутым магнитопроводом. Электричество, 1947, 2. [Shturman G. I., Aronov R. L. Edge effect in induction machines with an open magnetic circuit. Electricity, 1947, 2.] 
[2] Вольдек А. И. Продольный краевой эффект во вторичной цепи индукционных машин и насосов для жидких металлов с разомкнутым магнитопроводом. Известия вузов, Электромеханика, 1960, 3. [Vol'dek A. I. Longitudinal edge effect in the secondary circuit of induction machines and pumps for liquid metals with an open magnetic circuit. News of high schools, Electromechanics, 1960, 3.]

[3] Вольдек А. И. Индукционные магнитогидродинамические машины с жидкометаллическим рабочим телом. Л.: Энергия, 1970. С. 249. [Vol'dek A. I. Induction magnetohydrodynamic machines with liquid metal working fluid. L., Energy, 1970. P. 249.]

[4] Гринберг Г.А. Избранные вопросы математической теории электрических и магнитных явлений. Л.: Издательство АН СССР, М., 1948. 727 с. [Grinberg G.A. Selected problems of mathematical theory of electrical and magnetic phenomena. Moscow, USSR Academy of Sciences, 1948,727 p.] 